\title{
Effectiveness of Activity-Based Therapy in Comparison with Surface Spinal Stimulation in People with Traumatic Incomplete Spinal Cord Injury for Activation of Central Pattern Generator for Locomotion: Study Protocol for a 24-Week Randomized Controlled Trial
}

\author{
Parneet Kaur Bedi ${ }^{1}$, Narkeesh Arumugam ${ }^{1}$, Harvinder Singh Chhabra ${ }^{2}$ \\ ${ }^{1}$ Department of Physiotherapy, Punjabi University, Patiala, India \\ ${ }^{2}$ Department of Spine Services, Indian Spinal Injuries Centre, New Delhi, India
}

\begin{abstract}
Study Design: A multi-centric randomized controlled trial to be conducted at two sites, department of physiotherapy, Punjabi University, Patiala and rehabilitation department, Indian Spinal Injury Centre, New Delhi, India.

Purpose: To determine the effectiveness of activity-based therapy in comparison with surface spinal stimulation (SSS) in traumatic incomplete spinal cord injury (SCI) with special reference to locomotion - a central pattern generator controlled function.

Overview of Literature: A major goal for many patients after SCI is to regain the function of locomotion. It is crucial that rehabilitation strives to maximize locomotor ability and functional recovery after SCI. Experimental evidence of improvement in stepping and motor control after activity-based training in animal models and human SCl has been translated into clinical neuro-rehabilitation.

Methods: Control group participants will undertake an intensive 24-week duration thrice weekly program of activity-based therapy. In addition to this the participants in experimental group will also receive a session of 45 minutes of SSS on thrice weekly basis. The primary analysis for our study will be at 24 weeks. Linear regression will be used to determine the mean between-group differences and $95 \%$ confidence interval for all continuous outcomes using baseline scores and group allocation as covariates.

Results: The primary outcome measure is improvement in the level of walking index for SCI-II. The secondary outcome measures are modified Ashworth scale, Penn spasm frequency score, spinal cord independence measure-III, SCl functional ambulation inventory, Hoffman's reflex, somatosensory evoked potential, and American Spinal Injury Association Impairment Scale scores.

Conclusions: An insight into training-induced mechanisms will be of great importance to fine tune such combined treatments and vindicate their efficacy in restoration of locomotion and functional activities in individuals with SCI.
\end{abstract}

Keywords: Spinal cord injury; Central pattern generation; Locomotion; Activity based therapy; Surface spinal stimulation

Received Oct 3, 2017; Revised Nov 1, 2017; Accepted Nov 10, 2017

Corresponding author: Parneet Kaur Bedi

Punjabi University Faculty of Medicine, Physiotherapy Science Block 4, Punjabi University, Patiala, Punjab, 147002, India

Tel: +91-9888229595, Fax: +91-9888229595, E-mail: Parneet.bedi@yahoo.co.in 


\section{Introduction}

The ability to walk again is often the first goal mentioned by a person who has recently sustained a spinal cord injury (SCI), but it is usually the last goal to be addressed for those with motor complete injures [1]. A traumatic SCI is a lesion of neural elements of the spinal cord that can result in any degree of sensory and motor deficit, autonomic or bowel dysfunction.

The prognosis for regaining the ambulatory function in people with traumatic SCI ranges from 3\% (for the American Spinal Injury Association Impairment Scale [AIS] score A, i.e., complete injury) to $95 \%$ (AIS D, i.e., incomplete injury) [2]. It is reported that for patients with a motor complete and sensory incomplete SCI (AIS B) the chance to become ambulatory is $50 \%$. A major goal for many patients after SCI is to recover the ability to walk. It is crucial that rehabilitation strives to maximize locomotor ability and functional recovery after SCI. Experimental evidence of improvement in stepping and motor control after activity-based training in animal models and human SCI has been translated into clinical neuro-rehabilitation.

Many strategies exist to improve locomotion, such as treadmill training with and without body weight support, robotic-assisted gait training, functional electrical stimulation, epidural stimulation, and surface spinal stimulation (SSS) [3]. One of the recent methods for the management of incomplete spinal cord injuries in terms of functional recovery and locomotion include spinal stimulation for central pattern generator (CPG) [4]. CPG is defined as a neural circuit that can produce self-sustained patterns of behavior, independent of sensory input [5]. This network generates the rhythm and shapes the pattern of bursts of motoneurons [6,7]. Central pattern generation has been identified as an important component of the neural control of locomotion by the mammalian spinal cord. The generation of oscillatory flexor and extensor ventral root output from the neural circuits of the spinal cord in the absence of oscillatory input from the brain or from the periphery defines central pattern generation.

There are three interventions that have been proven successful in modulating the physiological state of the spinal circuits associated with successful postural and locomotor activity. The first intervention involves the modulation of the locomotor circuits through activity-dependent mechanisms. Several studies have shown that the spinal cord learns to perform the task that it practices. A second in- tervention involves modulation of the locomotor circuits pharmacologically. A third intervention is the modulation of the physiological state of the spinal circuitry via epidural stimulation. It seems highly probable that the chronic stimulation techniques presently used to suppress pain and spasticity can be readily adapted to facilitate postural and locomotor control [8].

Rehabilitative training is currently one of the most successful treatments to promote functional recovery following SCI. Nevertheless, there are many unanswered questions including the most effective and beneficial design, and the mechanisms underlying the training effects on motor recovery. Furthermore, the outcome of combination of electrotherapeutics and rehabilitative training on individuals with SCI needs to be analyzed. An insight into training-induced mechanisms will be of great importance to fine tune such combined treatments and vindicate their efficacy in restoration of locomotion and functional activities in individuals with SCI.

This clinical trial is an attempt to determine the changes in neural circuit in spinal cord after rigorous training and electrotherapeutics in frame of neural plasticity. The purpose of this study is to determine the effectiveness of activity-based therapy (ABT) in comparison with SSS in traumatic incomplete SCI with special reference to locomotion-a CPG controlled function.

\section{Materials and Methods}

A prospective, parallel pragmatic, multi-centric randomized controlled trial will be undertaken, in which people with traumatic incomplete SCI will be randomized into either the control group or the experimental group over 24 weeks.

\section{Participants}

Participants will be included if they: (1) have sustained a traumatic SCI a minimum of 6 months prior to consent and have completed their primary rehabilitation; (2) are 18 years or older and able to give informed consent; (3) are male and female subjects; (4) have a traumatic incomplete SCI (AIS C and D as per the AIS); (5) are able and willing to attend an exercise program 3 times per week for 24 weeks; (6) are considered by their medical consultant to be fit to undertake the exercise program; and (7) individual should be able to understand or follow commands 
and instructions.

Participants will not be included if they: (1) have brachial plexus, cauda equina, or peripheral nerve injury; (2) have had recent major trauma or surgery within the last 6 months; (3) have an existing stage 3 or 4 pressure ulcer according to the National Pressure Ulcer Advisory Panel classification; (4) are post-menopausal at the time of injury (females); (5) are having fixed joint deformity, severe spasticity; (6) are cases of non-traumatic SCI, space occupying lesion, and compressive myelopathies; (7) have presence of any cognitive deficit, or any other neurological disorder; (8) have a medical history of exposure to medication(s) known to affect mineral or bone metabolism; and (9) are likely to experience clinically significant autonomic dysreflexia and/or orthostatic hypotension in response to electrical stimulation or prolonged upright postures.

\section{Recruitment strategy and time frame}

This study seeks to enroll 40 patients with traumatic incomplete SCI. This assumes a standard deviation of 2.25 points (WISCI-II), loss to a follow-up of $15 \%, \alpha=0.05$, with $80 \%$ power, and a minimal worthwhile effect of 1 point.

The recruitment of study participants will take place from the outpatient department of department of physiotherapy, Punjabi University, Patiala, Punjab and rehabilitation department of Indian Spinal Injury Centre, New Delhi, India. If the patient meets the eligibility criteria for the study, the designated study personnel will obtain the informed consent and case report forms (CRFs).

The ABT program and SSS will be delivered by a wellqualified physiotherapist (research fellow). The research fellow of the present study will obtain data from the patient, family members, and medical record where available and will complete the CRF and record this information.

\section{Assignment of intervention}

A computer-generated random allocation will be formulated prior to commencement of the study by an independent person located off the study site. A blocked allocation (1:1) schedule will be used. Participants will be randomly allocated to one of the two groups using the principle of concealed allocation. Concealment will be ensured by placing each participant's allocation into a sealed, opaque, and sequentially numbered envelope. The envelopes will be kept off site by an independent person. Once a participant has completed the baseline assessment and his/her details are lodged, the researcher will contact the independent person to open an envelope and attain the participant's allocation. The participant will be considered to have entered the trial at this point. The research fellow will be delivering the interventions as per the assignment of the group. The principal investigator will be assessing the participants at the baseline, at 12 weeks, and at 24 weeks. The principal investigator will be blinded. The statistician conducting the data analysis will also be blinded to group allocation.

\section{Intervention}

Activity-based interventions or therapies include any therapy activity, or intervention, that is focused on improving muscle function and sensory perception below the level of injury, and not simply accommodation or compensation for the paralysis and sensory loss due to the SCI, in order to improve overall function after SCI [9].

SSS is non-invasive form of electrical stimulation delivered at the T10-L1 vertebral level with the adhesive electrodes placed para-vertebrally on each side of spine $5 \mathrm{~cm}$ apart. Electrodes are self-adhesive in nature rectangular $4.5 \mathrm{~cm} \times 9 \mathrm{~cm}$ in size. The electrical stimulations will have an amplitude modulated alternating current, with a carrier frequency of $2,500 \mathrm{~Hz}$, modulated to "beat" frequency of $20-70 \mathrm{~Hz}$ and stimulation amplitude will be raised to elicit sensory stimulation [10].

Control group participants will undertake an intensive 24-week duration thrice weekly program of ABT. In addition to ABT the participants in experimental group will also receive a session of 45 minutes of SSS on thrice weekly basis (Fig. 1).

The exercises for the participant were designed according to the principles of ABT. Exercises were determined according to the requirement of the participant. Session of ABT will be according to the physical capacity of the participant (3-4 hours). It will also include locomotor training using Body weight support treadmill training (BWSTT) for thirty minutes to one hour in each ABT session. In this procedure a portion of the patient's body weight is supported while the patient is assisted to walk on a motorized treadmill with the goal of providing normal kinematic and temporal cues during walking. BWSTT 


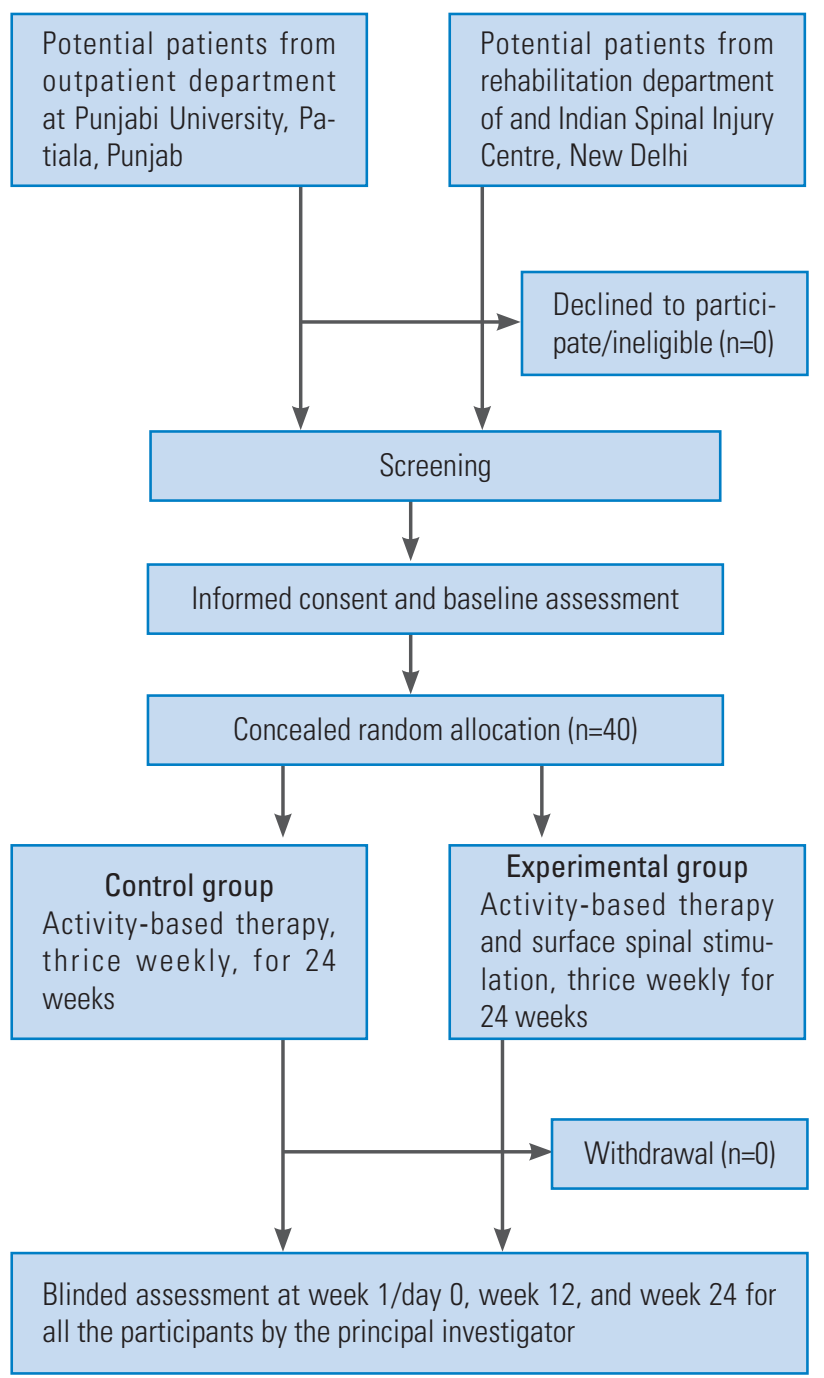

Fig. 1. Flow diagram of the study protocol.

is based on practicing a normal physiologic gait pattern, with attention to the ideal kinematic and temporal aspects of gait [11].

This study will follow the ABT guidelines depicting the principles [9].

\section{1) Phase I/II}

Reactivation/re-organization and development/stabilization phase: stimulate the nervous system with active assisted exercises and use developmental sequencing to develop joint stabilization.

\section{2) Phase III}

Strength: initiate eccentric and concentric muscle contractions through positional movement or stimulation.

\section{3) Phase IV}

Function and co-ordination: improve co-ordinated movement through all planes of movement and motion. Most exercises are performed in load bearing position (mainly free standing).

\section{4) Phase V}

Gait training: focus on proper gait mechanics and the ability to move over ground in multiple planes of motion.

\section{Data management}

Patient names and other direct identifiers will not appear on the CRFs. A master sheet will be used with identifying information during the enrolment period in order to avoid double entries and mistakes. All the CRF sheets will be checked for missing data and then the data will be recorded.

\section{Trial management}

Under the direction of the principal investigator, the present study will be conducted at the designated sites. The research personnel shall manage the day to day activities of the study.

\section{Ethical considerations}

This study will be conducted according to the international standards of good clinical practice, applicable government regulations and applicable institutional research policies and procedures as relevant to the study design. Research personnel will approach the eligible participants and they will sign an informed consent form to indicate their willingness to participate in this study. Confidentiality will be maintained throughout the study.

Ethical approval was obtained from the Institutional Ethics Committee of Punjabi University Patiala, Punjab and Indian Spinal Injury Centre, New Delhi. The results of this study will be disseminated through publications and presented at national and international conferences (clinical trial registry of India REF/2017/08/014989).

\section{Data integrity and management}

The data will remain confidential throughout the course 
of the study. Initially, data will be collected on paperbased CRFs. The paper-based CRFs will be compiled in participant-specific folders in the office of the principal investigator. Data will be managed and transcribed into electronic format.

\section{Withdrawal}

The study will have the following categories of participant withdrawals: withdrawal from the entire study with revocation of consent: no further follow-up or data collection will occur; lost to follow-up: the participant is lost to follow-up after baseline assessment. This may occur due to non-compliance of the participant with the requirements to follow the protocol.

\section{Data analysis}

All data will be analysed using IBM SPSS software ver. 22.0 (IBM Corp., Armonk, NY, USA) at the department of physiotherapy, Punjabi University, Patiala. A separate analysis will be done for all outcomes. The primary analysis for our study will be at 24 weeks. Linear regression will be used to determine the mean between-group differences and $95 \%$ confidence intervals for all continuous outcomes using baseline scores and group allocation as covariates. Missing data will not be imputed. All $p$-values less than 0.05 will be considered significant.

Table. 1. Time schedule of enrolment, intervention, and assessment of participant during the study

\begin{tabular}{|c|c|c|c|c|c|c|}
\hline \multirow{2}{*}{ Visit type } & \multirow{2}{*}{$\frac{\text { Enrolment }}{\text { Day }-7 \text { to } 1}$} & \multirow{2}{*}{$\frac{\text { Allocation }}{\text { Day 0/week } 1}$} & \multicolumn{3}{|c|}{ Post allocation } & \multirow{2}{*}{ End of study } \\
\hline & & & Week 1 & Week 12 & Week 24 & \\
\hline Protocol activity & $\sqrt{ }$ & & & & & \\
\hline Eligibility screening & $\sqrt{ }$ & & & & & \\
\hline Patient information sheet & $\sqrt{ }$ & & & & & \\
\hline Informed consent & $\sqrt{ }$ & & & & & \\
\hline Demographic details & $\sqrt{ }$ & & & & & \\
\hline Characteristics of SCl & $\sqrt{ }$ & & & & & \\
\hline Medical history & $\sqrt{ }$ & & & & & \\
\hline AIS assessment (ISNSCI) & $\sqrt{ }$ & & & & & \\
\hline Allocation & & $\sqrt{ }$ & & & & \\
\hline \multicolumn{7}{|l|}{ Intervention } \\
\hline Intervention group & & & $\sqrt{ }$ & $\sqrt{ }$ & $\sqrt{ }$ & \\
\hline Control group & & & $\sqrt{ }$ & $\sqrt{ }$ & $\checkmark$ & \\
\hline \multicolumn{7}{|l|}{ Assessment } \\
\hline MAS & & & $\sqrt{ }$ & $\sqrt{ }$ & $\sqrt{ }$ & \\
\hline Penn spasm frequency & & & $\sqrt{ }$ & $\sqrt{ }$ & $\sqrt{ }$ & \\
\hline SCIM-III & & & $\sqrt{ }$ & $\sqrt{ }$ & $\sqrt{ }$ & \\
\hline WISCI-II & & & $\sqrt{ }$ & $\sqrt{ }$ & $\sqrt{ }$ & \\
\hline SCI-FAI & & & $\sqrt{ }$ & $\sqrt{ }$ & $\sqrt{ }$ & \\
\hline Hoffman's reflex & & & $\sqrt{ }$ & $\sqrt{ }$ & $\sqrt{ }$ & \\
\hline SSEP & & & $\sqrt{ }$ & $\sqrt{ }$ & $\sqrt{ }$ & \\
\hline AIS assessment (ISNSCI) & & & $\sqrt{ }$ & $\sqrt{ }$ & $\sqrt{ }$ & \\
\hline Adverse effects & & & $\sqrt{ }$ & $\sqrt{ }$ & $\sqrt{ }$ & $\sqrt{ }$ \\
\hline
\end{tabular}

SCI, spinal cord injury; AIS, American Spinal Injury Association Impairment Scale score; ISNSCl, international standards for neurological classification of spinal cord injury; MAS, modified Ashworth scale; SCIM-III, spinal cord independence measure-III scale; WISCI-II, walking index for spinal cord injury-II; SCI-FAI, spinal cord injury functional ambulation inventory; SSEP, somatosensory evoked potential. 


\section{Results}

\section{Outcome measures}

All assessment will be conducted at the beginning, i.e., day $0 /$ week 1 , at 12 th week, and at 24 th week of the program. The assessment of the participants will be done by the principal investigator (Table 1).

\section{1) Primary outcome}

The primary outcome is the level of walking on WISCIII score sheet. Walking index for SCI assesses the amount of physical assistance needed as well as devices required for walking following paralysis that result from SCI. It has been designed to be a more precise measure of improvement in functional limits. The International Classification of Functioning, Disability and Health (ICF) domain is activity [12]. There are 20 levels on the scale ranged between $0-20$. Higher the score on the WISCI-II scale, better is the outcome.

\section{2) Secondary outcomes}

There will be seven secondary outcome measures. The details of each are as follows.

\section{(1) Modified Ashworth scale}

It measures spasticity in patients with lesions of the central nervous system. Modified Ashworth scale uses 6 point scale (range, 0 to 4 ). The ICF domain is body structure and body function [13]. Lower score on the scale means better outcome.

\section{(2) Penn spasm frequency scale}

A self-report measure assesses a patient's perception of spasticity frequency and severity. It is composed of two parts: the first is a self-report measure with items on 5-point scales developed to augment clinical ratings of spasticity and provides a more comprehensive assessment of spasticity. If the patient indicates no spasms in part 1 , then they do not proceed to part 2. The second component of the Penn spasm frequency scale is a 3-point scale assessing the severity of spasms [14]. Lower score on the scale means better outcome.

\section{(3) American Spinal Injury Association Impairment Scores}

The AIS is a multi-dimensional approach to categorize motor and sensory impairment in individuals with SCI. It identifies sensory and motor levels indicative of the most rostral spinal levels demonstrating "unimpaired" function. A 5-point ordinal scale, based on the Frankel scale, classifies individuals from "A" (complete SCI) to "E" (normal sensory and motor function). The present study will consider individuals with AIS C and AIS D. AIS C (motor incomplete): motor function is preserved below the neurological level and more than half of key muscle functions below the single neurological level of injury (NLI) have a muscle grade less than 3. AIS D (motor incomplete): motor function is preserved below the neurological level and at least half of key muscle functions below the NLI have a muscle grade of 3 or greater (https://www.scireproject. com) [15].

\section{Spinal cord independence measure}

It is the only comprehensive functional rating scale designed specifically for patients with SCI that measures independence in all aspects of primary daily activities relevant for patients with SCL, scores independence in performing various tasks according to their value for the patient, and scores every task or area of function according to its relative weight in the total relevant daily function. The ICF domain is activity [16]. The total score for the scale is 100 points. Higher score on the spinal cord independence measure-III scale means better outcome.

\section{Spinal cord injury functional ambulation inventory}

This scale is an observational gait assessment that includes three key domains of walking functions. Maximum score of the scale is 39 points. Higher scores denote better levels of function in each subscale. The subscales include: gait parameters, assistive devices, and temporal parameters [17].

\section{Somatosensory evoked potential}

This study evaluates time-locked responses of the nervous system to an external stimulus. They represent the function of the ascending sensory pathways using an afferent potential, which travels from the peripheral nerve to the plexus, root, spinal cord (posterior column), contralateral medial lemniscus, thalamus, to the somatosensory cortex. It is initiated by a repetitive submaximal stimulation of 
a sensory nerve, mixed nerve, or dermatome and is recorded from the spine or scalp [18]. The present study will consider somatosensory evoked potential of tibial nerve for lower limb.

\section{Hoffman's reflex}

It is a monosynaptic reflex elicited by submaximal stimulation of the tibial nerve and recorded from calf muscles. It evaluates the proximal sensory and motor pathways and is commonly used in studies relating to central nervous system disorders [19].

\section{Patient safety and confidentiality}

There are no anticipated adverse events and risks to the safety of the patient. All patients will have a numeric identification number. Patient names and other direct identifiers will not appear on the CRFs. Data analysis will be conducted on de-identified data and only group data will be presented. The participants may benefit from the regimen of the intervention which may be above standard of care.

\section{Discussion}

This randomized controlled trial will examine the efficacy of ABT in comparison to SSS on functional outcomes and locomotion in individuals with incomplete SCI. This will be one of the first of its kind study involving people with SCI from in developing country. Assessors will be blinded, but it is not possible to blind participants or the clinicians.

\section{Conclusions}

This study shall provide insight into the therapeutic regimen that can be most effective, non invasive, and beneficial, to tap the spinal neural circuitry mechanisms for motor and functional recovery in individuals with incomplete spinal cord injury.

\section{Conflict of Interest}

No potential conflict of interest relevant to this article was reported.

\section{References}

1. Chafetz R, Johnston T, Calhoun C. Outcomes in upright mobility in individuals with a spinal cord injury. Top Spinal Cord Inj Rehabil 2005;10:94-108.

2. Wirz M, Bastiaenen C, de Bie R, Dietz V. Effectiveness of automated locomotor training in patients with acute incomplete spinal cord injury: a randomized controlled multicenter trial. BMC Neurol 2011;11:60.

3. Mehrholz J, Kugler J, Pohl M. Locomotor training for walking after spinal cord injury. Cochrane Database Syst Rev 2008;(2):CD006676.

4. MacKay-Lyons M. Central pattern generation of locomotion: a review of the evidence. Phys Ther 2002;82:69-83.

5. Grillner S. Interaction between sensory signals and the central networks controlling locomotion in lamprey, dogfish and cat. In: Grillner S, Stein PS, Stuart DG, Forssberg H, Herman RM, editors. Neurobiology of vertebrate locomotion. London: Palgrave Macmillan; 1986. p.505-12.

6. Grillner S, Deliagina T, Ekeberg O, et al. Neural networks that co-ordinate locomotion and body orientation in lamprey. Trends Neurosci 1995;18:270-9.

7. Grillner S. Neurobiological bases of rhythmic motor acts in vertebrates. Science 1985;228:143-9.

8. Behrman AL, Harkema SJ. Physical rehabilitation as an agent for recovery after spinal cord injury. Phys Med Rehabil Clin N Am 2007;18:183-202.

9. Jones ML, Harness E, Denison P, Tefertiller C, Evans $\mathrm{N}$, Larson CA. Activity-based therapies in spinal cord injury: clinical focus and empirical evidence in three independent programs. Top Spinal Cord Inj Rehabil 2012;18:34-42.

10. Wang RY, Chan RC, Tsai MW. Effects of thoracolumbar electric sensory stimulation on knee extensor spasticity of persons who survived cerebrovascular accident (CVA). J Rehabil Res Dev 2000;37:73-9.

11. Bedi PK, Arumugam N. Activity based therapy and surface spinal stimulation for recovery of walking in individual with traumatic incomplete spinal cord injury: a case report. Int J Recent Sci Res 2015;6:55813.

12. Bohannon RW, Smith MB. Interrater reliability of a modified Ashworth scale of muscle spasticity. Phys Ther 1987;67:206-7. 
13. Penn RD, Savoy SM, Corcos D, et al. Intrathecal baclofen for severe spinal spasticity. N Engl J Med 1989;320:1517-21.

14. Noonan V, Mak J, Zhu J, Diab K, Queree M. American Spinal Injury Association Impairment Scale (AIS): international standards for neurological classification of spinal cord injury (ISNCSCI) [Internet]. Vancouver (BC): The SCIRE Project; 2016 [cited 2017 Oct 29]. Available from: https://scireproject. com/outcome-measures/outcome-measure-tool/ american-spinal-injury-association-impairmentscale-ais-international-standards-for-neurologicalclassification-of-spinal-cord-injury.

15. Dittuno PL, Ditunno JF Jr. Walking index for spinal cord injury (WISCI II): scale revision. Spinal Cord 2001;39:654-6.
16. Catz A, Itzkovich M, Agranov E, Ring H, Tamir A. SCIM: spinal cord independence measure: a new disability scale for patients with spinal cord lesions. Spinal Cord 1997;35:850-6.

17. Field-Fote EC, Fluet GG, Schafer SD, et al. The spinal cord injury functional ambulation inventory (SCIFAI). J Rehabil Med 2001;33:177-81.

18. Freeman TL, Johnson E, Freeman ED, Brown DP. Somatosensory evoked potentials (SSEP). In: Cuccurullo SJ, editor. Physical medicine and rehabilitation board review. New York (NY): Demos Medical Publishing; 2004. p.326-7.

19. Mishra UK, Kalita J. Clinical neurophysiology. 2nd ed. Reed: Elsevier; 2008. 